Original

\title{
Análisis retrospectivo de la continencia urinaria en pacientes sometidos a braquiterapia post-resección transuretral prostática
}

\author{
Daniel Ramirez Claros, João Antonio Pereira Correia, Luiz Renato Muglia Ferreira, \\ Fernando Pires Vaz \\ Departamento de Urología. Hospital dos Servidores do Estado do Rio de Janeiro. Brasil.
}

\begin{abstract}
Resumen
Introducción y objetivos: Algunos de los pacientes candidatos a la braquiterapia presentan historia previa de resección transuretral de la próstata (RTUP). La RTUP, sin embargo, crea una cavidad que puede llevar a algunas dificultades técnicas para la realización de braquiterapia. Tales condiciones tornan a la RTUP, previa a la braquiterapia, una contraindicación relativa a la realización de ésta.

El objetivo del presente trabajo es evaluar las tasas de incontinencia urinaria en pacientes submetidos a braquiterapia despues de una RTUP por medio de análisis retrospectiva.

Materiales y métodos: En nuestro estudio analizamos 16 pacientes sometidos a RUTP previa a braquiterapia. Tras braquiterapia los pacientes fueron evaluados sobre síntomas del tracto urinario inferior a través del cuestionario ICSmaleSF. $\mathrm{El}$ intervalo de tiempo entre la RTUP y el tratamiento braquiterápico varió de 30 a 90 días, (media= 60 días). El seguimiento post braquiterapia varió entre 3 a 60 meses, (media $=30$ meses).

Resultados: Ninguno de los 16 pacientes evaluados presentó síntomas del tracto urinario inferior o incontinencia urinaria. Con el cuestionario ICSmaleSF la puntuación de interferencia en la calidad de vida obtuvo una media de 0,18 antes de la braquiterapia (variando de 0 a 1) y después de la braquiterapia de 0,20 (variando de 0 a 1) (p<0,001), donde las diferencias de los resultados no fue estadísticamente significativa. Algunas complicaciones fueron observadas como: disfunción eréctil, equimosis escrotal y retención urinaria. Los casos de disuria, equimosis escrotal y retención urinaria fueron de corta duración y resolución espontánea, éste último tras cateterismo vesical por 7 días. El paciente portador de disfunción eréctil presentó buena respuesta al uso semanal (3x/semana) de inhibidor de fosfodiesterasa tipo 5 , después de un periodo de 6 meses.

Conclusión: En concordancia con otros estudios, nosotros creemos que con alguna estrategia la RTUP no debería ser considerada una contraindicación relativa para la braquiterapia.
\end{abstract}

Palabras clave: Braquiterapia. Resección transuretral de próstata. Neoplasia prostática. Radioterapia.

\section{Retrospective evaluation of urinary continence in patients submitted to brachytherapy after transurethral resection of the prostate gland}

\section{Abstract}

Introduction and Objective: Some candidates for brachytherapy present with a previous history of prostate transurethral resection (TURP). Nonetheless, TURP generates a cavity that may lead to technical difficulties in performing brachytherapy. Such condition make a history of previous TURP a relative contra-indication for brachytherapy. The aim of this study is to evaluate the role of brachytherapy in urinary continence, in a group of patients with previous history of TURP.

Materials and Methods: In our study, we analysed a group of 16 patients submitted to TURP pre-brachytherapy. The mean quality of life score from ICSmaleSF questionnaire ranged from 0 to 1 before and after brachytheraphy (average: 0,18 -before; average: 0,20 - after). There was no estatistically significant difference between the former values ( $<<0,001)$. After brachytherapy patients were evaluated for lower urinary tract symptoms. The time interval between TURP and brachytherapy ranged from 30 to 90 days (average: 60 days). Post-brachytherapy follow-up varied between 3 and 60 months (average: 30 months).

Results: None of the 16 patients evaluated presented lower urinary tract symptoms nor urinary incontinence. Medical complications observed in patients were: dysuria, erectile dysfunction, scrotal ecchymosis and urinary retention . The cases of dysuria, scrotal ecchymosis and urinary retention observed were of short-duration, the last one with spontaneous resolution after 7 days of vesical catheterism. The patient presenting erectile dysfunction had a good response to oral type 5 fosfodiesterase inhibitor three times a week, after a six month period.

Conclusion: In accordance with other studies, we believe that with some strategy TURP should not be considered a relative contra-indication for brachytherapy.

Keywords: Brachytherapy. Transurethral resection of prostate. Prostatic. Neoplasms. Prostate. Radiotherapy. 
T a braquiterapia prostática de baja tasa de dosis, Uutilizando como elemento radioactivo el Iodo125 o el Palladium 103, está cada vez mas empleado como opción terapéutica en individuos portadores de carcinoma de próstata confinado a la glándula y enfermedad de bajo volumen ${ }^{1}$, así como en estadios iniciales ${ }^{2}$.

Algunos de los pacientes candidatos a la braquiterapia presentan historia previa de resección transuretral de la próstata (RTUP) ${ }^{1}$, en función de los síntomas obstructivos del tracto urinario inferior, consecuente de hipertrofia benigna, o por la misma proliferación neoplásica del carcinoma prostático.

La RTUP, sin embargo, crea una cavidad que puede llevar a dificultades técnicas para la realización de la braquiterapia, como por ejemplo: dificultades en la fijación de las semillas radioactivas al tejido prostático; aumento en la posibilidad de incontinencia urinaria (mayor proximidad de las semillas al músculo esfínter uretral externo); mala distribución de la dosis radioactiva (el "defecto uretral” así creado por la RTUP es asimétrico e irregular, con subsiguiente mal alineamiento de las agujas y dificultad de homogeneización de la dosis en comparación con la glándula entera) ${ }^{1}$. Tales condiciones tornan a la RTUP, previa a la braquiterapia, una contraindicación relativa a la realización de ésta ${ }^{3,4}$.

En nuestro estudio analizamos un grupo de pacientes sometidos a RTUP previa a braquiterapia.

\section{MATERIALES Y MÉTODOS}

En el periodo de 2001 a 2008 fueron analizados 16 pacientes tratados con RTUP previa a la braquiterapia, con edades comprendidas entre 73 y 82 años de edad (edad media -78 años). De éstos, 2 fueron sometidos previamente a prostatectomía a cielo abierto. Las co-morbilidades del grupo están expuestas en la Tabla 1.

Tabla 1. Co-morbilidades de los pacientes sometidos a RTUP pre-braquiterapia

\begin{tabular}{lc}
\hline Co-morbilidades & n (\%) \\
\hline Enfermedad de Paget & $1(6,25)$ \\
Cardiopatía hipertensiva & $2(12,5)$ \\
Hipertensión & $1(6,25)$ \\
Diabetes & $2(12,5)$ \\
Diabetes y Parkinson & $1(6,25)$ \\
Sin co-morbilidades & $9(56,25)$ \\
\hline
\end{tabular}

Todos los pacientes fueron evaluados por un equipo multidisciplinar formado por Urólogos, Oncologistas radioterapeutas y Patólogos, envueltos con la investigación y tratamiento del cáncer de próstata. Los pacientes fueron evaluados a través del examen físico con el tacto rectal, dosificación sérica del PSA total pre y post braquiterapia, biopsia prostática y ecografía transrectal con frecuencia de 5,0 a 7,5 $\mathrm{MHz}$, preferencialmente biplanar, para determinar la configuración del implante, numero de las semillas radioactivas y su localización en la próstata.

En virtud del elevado volumen prostático, 7 pacientes fueron sometidos a terapia hormonal prebraquiterapia, por 6 meses, utilizando fármaco análogo de LHRH (acetato de leuprolida), de acuerdo con protocolos previamente establecidos ${ }^{5}$. El volumen glandular en estos pacientes variaba de $44 \mathrm{~g}$ a $74 \mathrm{~g}$, con media de $59 \mathrm{~g}$, con reducción para un corte de 15 g a 45 g, con media de 34 g.

En los pacientes donde no fue necesario reducción dimensional para la braquiterapia, el volumen prostático variaba de 20 g a 36 g, con media de 23 $\mathrm{g}$, fue realizado RTUP para poder disminuir la dosis de irradiación en la uretra y eliminar los síntomas del tracto urinario inferior.

El valor medio del PSA total sérico, antes del tratamiento radioactivo y hormonal (cuando necesario), variaba de $4 \mathrm{ng} / \mathrm{ml}$ a $19 \mathrm{ng} / \mathrm{ml}$, con media de $5,79 \mathrm{ng} / \mathrm{ml}$. Al término de la braquiterapia la media de PSA disminuyó para $0,1 \mathrm{ng} / \mathrm{ml}$, con PSA total sérico máximo equivalente a $0,61 \mathrm{ng} / \mathrm{ml}$. La evaluación histopatológica del sistema de graduación de Gleason evidenció la distribución que sigue en la Tabla 2 .

De acuerdo con la clasificación de riesgo (en la que fue evaluado el estadio clínico, el grado de Gleason y el PSA pre-braquiterapia) fue encontrado que 2 pacientes eran de riesgo intermediario y 14 eran de bajo riesgo. El estadio clínico varió de $\mathrm{T} 1 \mathrm{a}$ T2b.

Tabla 2. Sistema de Graduación de Gleason presentada en el estudio histopatológico prostático, de los pacientes sometidos a la braquiterapia tras RTUP

\begin{tabular}{lc}
\hline Sistema de Gleason & n (\%) \\
\hline G4 $(2+2)$ & $1(6,25)$ \\
G6 $(3+3)$ & $12(75,0)$ \\
G7 $(3+4)$ & $2(12,5)$ \\
Gleason no aplicable (G0) & $1(6,25)$ \\
\hline
\end{tabular}


Para la realización de la braquiterapia fueron incluidos pacientes con histopatológico Gleason variando de 4-7 con o sin terapia hormonal previa ${ }^{6}$.

El implante fue realizado por dos de los Urólogos del equipo multidisciplinar especializados en braquiterapia prostática.

La dosis radioactiva utilizada fue según las normas establecidas por la American Association of Physics and Medicine (AAPM) Task Group no. 43 (TG-43) ${ }^{7}$. Para la dosimetría fue utilizado el sistema D90 que variaba entre $(102,36 \%$ a $134,23 \%)$ encontrándose una media de $120,025 \%$. El V100 de la próstata variaba entre $(90,28 \%$ a $99,26 \%)$ con una media de $95,621 \%$ y el V150 de la uretra variaba entre $(0 \%$ a $0,9 \%)$ con una media de $0,3 \%$. El tipo de ecografía utilizado fue 3D transrectal en tiempo real.

Al término de la braquiterapia los pacientes fueron evaluados para la sintomatología del tracto urinario inferior a través del cuestionario ICSmaleSF${ }^{1}$.

$\mathrm{El}$ intervalo de tiempo entre la RTUP y el tratamiento braquiterápico varió de 30 a 90 días, con media de 60 días. El seguimiento post braquiterapia varió entre 3 a 60 meses, con media de 30 meses.

\section{RESULTADOS}

De los pacientes evaluados ninguno presentó sintomatología referente al tracto urinario inferior de acuerdo con el cuestionario ICSmaleSF. Nuestra media de puntuación miccional fue de 0,43 (variando de 0 a 2), nuestra media de puntuación para la incontinencia fue de 0,18 (variando de 0 a 1) antes de la braquiterapia. Después de la braquiterapia los pacientes presentaron una media de "voiding score" de 0,5 (variando de 0 a 2) y nuestra media de puntuación para la incontinencia fue de 0,16 (variando de 0 a 1). La puntuación de interferencia en la calidad de vida obtuvo una media de 0,18 antes de la braquiterapia (variando de 0 a 1 ) y después de la braquiterapia de 0,20 (variando de 0 a 1$)(p<0,001)$, donde las diferencias de los resultados no fue estadísticamente significativa. Ninguno de los pacientes presentó nicturia antes y después de la braquiterapia y todos presentaban frecuencia urinaria a cada 4 horas, o más.

Las complicaciones observadas por los pacientes sometidos a braquiterapia al término de la RTUP están expuestas en la Tabla 3. Ninguno de los pacientes presentó incontinencia urinaria.

En cuanto a la disuria, la equimosis escrotal y la retención urinaria observadas, fueron de corta dura-
Tabla 3. Complicaciones póst-braquiterapia

\begin{tabular}{lc}
\hline Complicaciones & n (\%) \\
\hline Disúria & $2(12,5)$ \\
Equimosis escrotal & $1(6,25)$ \\
Retencion urinaria & $2(12,5)$ \\
Disfunción eréctil & $1(6,25)$ \\
\hline
\end{tabular}

ción, con resolución espontánea de ésta última después del cateterismo vesical por 7 días. El paciente portador de disfunción eréctil presentó buena respuesta al uso semanal (3x/semana) de inhibidor de fosfodiesterasa tipo 5 , después de un periodo de 6 meses.

\section{DISCUSIÓN}

La incontinencia urinaria post-braquiterapia es un síntoma que se limita, en la mayoría de los casos, a pérdidas esporádicas y reducidas. La pérdida de mas de algunas gotas, pérdida continua diaria o el uso de absorbentes femeninos, alcanzaron apenas $11 \%, 11 \%$ y $16 \%$ de los pacientes respectivamente, después de análisis de 105 pacientes, realizado por Talcott y colaboradores ${ }^{9}$. Considerando apenas la RTUP observamos tasas de incontinencia urinaria después del procedimiento, variando entre 1 a $5 \%^{6}$. Cuando asociadas la RTUP y la braquiterapia, presentan efectos colaterales sumatorias, sobre el tracto urinario inferior. La predisposición para los disturbios de la continencia miccional por la radiación es probablemente explicada por el comprometimiento de los esfínteres urinarios y la exposición del lecho prostático, consecuentes de la resección. Entretanto, las tasas de incontinencia urinaria en pacientes sometidos a braquiterapia, tras RTUP, todavía son muy variables, sin existencia de un consenso. Al revisar los estudios sobre el tema, observamos que éstas tasas pueden variar de 0 a $70 \%{ }^{10-16}$. Un análisis reciente de 118 pacientes, divulgada en un encuentro científico de la Sociedad Americana de Terapia Radiológica y Oncológica (ASTRO), Moran, refiere tasas de incontinencia urinaria variando de 7 a $24 \%$, valores semejantes a los estudios anteriores.

Abascal Junquera y colaboradores obtuvieron una tasa de incontinencia de $0,2 \%$ en 14 pacientes con RTUP previa ${ }^{17}$. En nuestro estudio no observamos incontinencia urinaria en ninguno de los pacientes, así como observado por Stone $^{18}$ en 43 
pacientes sometidos a RTUP previa a la braquiterapia, donde el implante radioactivo a base de Iodo125, en tiempo real, fue realizado en la periferia de la glándula, respetando distancia de $5 \mathrm{~mm}$. de la luz uretral, en un seguimiento de 18 meses.

Creemos que algunos factores como la presencia de la incontinencia urinaria previa a la braquiterapia, consecuente exclusivamente de la resección prostática, deben ser meticulosamente considerados, para evitar conclusiones no verdaderas sobre los efectos de la radioterapia. Como ejemplo, citamos el análisis de pacientes tratados en el Instituto de Radiología de Mallinckrodt, donde Pérez y colaboradores ${ }^{19}$ reportaron aumento de la incontinencia urinaria en pacientes tratados con RTUP previa. Subsecuentemente, en un análisis mas detallado de esos pacientes, los mismos autores rectificaron los resultados preliminares ya que los pacientes presentaban algún grado de incontinencia consecuente de la RTUP, mismo antes de la braquiterapia.

De acuerdo con las teorías propuestas por Wallner y colaboradores ${ }^{6}$ creemos que la principal explicación para la manutención de la continencia urinaria en nuestro estudio esté relacionada con la menor dosis de radiación uretral, una vez que las semillas radioactivas fueron implantadas, desde el inicio, lo mas distante posible del centro de la uretra. La distancia precisa del implante a partir de la uretra, depende parcialmente del tamaño de la próstata, pero generalmente es de $1 \mathrm{~cm}$. o lo mas distante posible. Dattolli y colaboradores ${ }^{20}$ relatan un margen de 0,5 a $1 \mathrm{~cm}$. en los diámetros transverso, antero-posterior y longitudinal. Las recomendaciones propuestas por la Sociedad Americana de Braquiterapia también sugieren que la técnica de implantes periféricos tiende a reducir las altas dosis en el centro de la glándula prostática y disminuyen la morbilidad urinaria ${ }^{7,21}$.

Una segunda hipótesis para la ausencia de incontinencia en nuestra serie es la relacionada a la técnica intra-operatoria de la RTUP. Fueron excluidos pacientes con defectos uretrales muy extensos una vez que tales defectos limitan la cantidad de tejido para la fijación de las semillas. En concordancia, Blasko y colaboradores ${ }^{13}$ recomiendan como criterio de implante, la manutención de por lo menos $1 \mathrm{~cm}$ de tejido prostático alrededor de la uretra.

El intervalo mínimo de 2 meses entre la RTUP y la implantación radioactiva, permitiendo, hipotéti- camente, la cicatrización uretral, también puede funcionar como teoría para justificar el mantenimiento de la continencia urinaria. Seymore y colaboradores $^{22}$, analizaron 368 pacientes sometidos a la braquiterapia tras RTUP, en el periodo de 1975 a 1982, que desarrollaron estenosis de uretra y estenosis del cuello vesical, y sugieren que el tiempo de 4 a 6 semanas es el intervalo apropiado entre la cirugía y la irradiación. El tiempo de espera de 12 semanas para realizar el implante fue efectuado por Abascal Junquera y colaboradores obteniendo escasa clínica obstructiva con una tasa de incontinencia de $0,2 \%{ }^{17}$.

Así como Moran y colaboradores ${ }^{23}$ proponen, creemos que, en casos seleccionados, no hay contraindicación para realización de braquiterapia tras RTUP, en virtud del bajo impacto asociado a la función urinaria. De la misma forma, Stone y Stock ${ }^{24}$, en un estudio conducido con 665 pacientes, recomiendan la braquiterapia en tiempo real, con implante de las semillas en la periferia lejos de la uretra. Del grupo inicial apenas 2 pacientes desarrollaron incontinencia urinaria, con seguimiento a largo plazo.

En relación al método ecográfico, cabe resaltar la evaluación realizada por Moran y colaboradores $^{25}$, que analizó 1959 pacientes sometidos a la braquiterapia de próstata, donde 152 presentaban previa RTUP, con media de seguimiento de 16 meses. Los pacientes eran evaluados sobre la presencia de cualquier pérdida urinaria. Después del análisis los autores proponen que usando una preplanificación en 3D y colocación periférica de las semillas, no hay contra-indicación para la realización de la braquiterapia pos-RTUP. Un "follow up" anticipado demostró una tasa inicial de incontinencia de $11 / 119$ pacientes $(9,2 \%)$. Si una definición exacta de incontinencia; incontinencia tiene que ser mayor a 1 pañal por día, la tasa de incontinencia cae para 4/119 pacientes $(3,4 \%)$. Nuestro estudio utilizó ecografía transrectal con frecuencia de 5,0 a 7,5 $\mathrm{MHz}$, biplanar, para determinar la configuración del implante, número de semillas y su localización en la próstata, obteniéndose el adecuado volumen planificado a ser tratado, no presentando consecuencias.

Creemos que el tema merece todavía mas discusiones donde estudios analizando un número mayor de pacientes será de gran utilidad para esclarecer esta afirmativa. 


\section{CONCLUSIÓN}

Nosotros creemos que con alguna estrategia, tal como una dosis baja de radiación en la uretra, implantes periféricos, defectos uretrales pequeños y 2 meses de intervalo mínimo entre RTUP y los implantes radioactivos, la RTUP para pacientes con síntomas del tracto urinario inferior, que son candidatos para la braquiterapia, puede ser indicado.

\section{REFERENCIAS}

1. Cesaretti JA, Stone NN, Stock RG. Does prior transurethral resection of prostate compromise brachytherapy quality: A dosimetric analysis. Int $\mathrm{J}$ Radiat Oncol Biol Phys. 2004 Oct 1; 60(2):648-653.

2. Aguiló Lucía F, Suárez Novo JF, Correa Generoso R, Boladeras Inglada AM, Polo Rubio A, Pera Fábregas J, et al. Estudio retrospectivo de 130 pacientes con cáncer prostático organoconfinado tratados con braquiterapia prostática. Actas Urol Esp. 2005; 29(1):47-54.

3. Dall'Oglio MF, Nadalin W, Vaz FP, Arruda HO, Silva ECCG. Câncer de próstata localizado: Tratamento. Brasilia: Associação Médica Brasileira e Conselho Federal de Medicina. Updated junho 2006. [citado em 27 de maio de 2008]. Available at http://www.projetodiretrizes.org.br/5_volume/04-Cancer.pdf

4. The American College of Radiology Practice Guideline for transperineal permanent brachytherapy of prostate cancer. American College of Radiology (ACR). 2005; pp. 909-15.

5. Abreu CEV, Nesrallah A, Silva JLF, Srougi M. Braquiterapia de câncer de próstata: Revisão e experiência inicial. Rev Soc Bras Cancer. 1999; 8: Artigo 36.

6. Wallner K, Lee H, Wasserman S, Dattoli M. Low risk of urinary incontinence following prostate brachytherapy in patients with a prior transurethral prostate resection. Int. J. Radiat Oncol Biol. Phys. 1997;37(3):565-569.

7. Nag S, Beyer D, Friedland J, Grimm P, Nath R. American Brachytherapy Society (ABS) recommendations for transperineal permanent brachytherapy of prostate cancer. Cancer. Int J Radiat Oncol Biol Phys 2004 Jul;44(4):789-799.

8. Donovan JL, Peters TJ, Abrams P, Brookes ST, de aa Rosette JJ, Schäfer W. Scoring the Short Form ICSmaleSF Questionnaire. International Continence Society. J Urol. 2000 Dec;164(6):19481955

9. Talcott JA, Clark JA, Stark PC, Mitchell SP. Long-Term Treatment Related Complications Of Brachytherapy For Early Prostate Cancer: A Survey Of Patients Previously Treated. J Urol 2001 Aug;166(2):494-499.

10. Dall'oglio MF, Nadalin W, Vaz FP, De Arruda HO, Silva ECCG. Tratamento Do Câncer Localizado Da Próstata. Int Braz J Urol 2004; 30 (Suppl. 1):21-29.

11. Green TC. Avances en Braquiterapia. Arch Esp Urol. 2005 May; 58(4):279-284.

12. $\mathrm{Hu} \mathrm{K}$, Wallner K. Urinary incontinence in patients who have a TURP/TUIP following prostate brachytherapy. Int J Radiat Oncol Biol Phys. 1998 Mar 1;40(4):783-786.
13. Blasko JC, Ragde H, Grimm PD. Transperineal ultrasound-guided implantation of the prostate: morbidity and complications. Scand J Urol Nephrol Suppl. 1991;137:113-118.

14. Gibbons RP, Mason JT, Correa RJ Jr, Cummings KB, Taylor WJ, Hafermann MD, Richardson RG. Carcinoma of the prostate: local control with external beam radiation therapy. J Urol. 1979 Mar; $121(3): 310-312$.

15. Moran B. Is Previous Transurethral Resection Of The Prostate A Contraindication To Brachytherapy? Annual Meetings of the American Society for Therapeutic Radiology and Oncology. 2002. Available at https://www.oncolink.org/conferences/ article. $\mathrm{cfm} ? \mathrm{c}=3 \& \mathrm{~s}=19 \& \mathrm{ss}=127 \& \mathrm{id}=660$.

16. Budía Alba A, Bosquet Sanz M, Tormo Micó A, Boronat Tormo F, Alapont Alacreu JM, Francés A, et al. Braquiterapia de baja tasa en el tratamiento del cáncer de próstata localizado. Actas Urol Esp. 2007;31(5):452-468.

17. Abascal Junquera JM, Hevia Suarez M, Abascal García JM, Abascal García R, Gonzalez Suárez H, Alonso A, et al. Braquiterapia en el cáncer de próstata. Actas Urol Esp. 2007;31(6): 617-626.

18. Stone NN, Ratnow ER, Stock RG. Prior transurethral resection does not increase morbidity following real-time ultrasound-guided prostate seed implantation. Tech Urol. 2000;6(2):123-127.

19. Perez CA, Walz BJ, Zivnuska FR, Pilepich M, Prasad K, Bauer W. Irradiation of carcinoma of the prostate localized to the pelvis: Analysis of tumor response and prognosis. Int $\mathrm{J}$ Radiat Oncol Biol Phys. 1980 May;6(5):555-563.

20. Dattoli MJ, Wallner K, Sorace R, Ting J. Planned extracapsular Seed Placement Using Palladium-103 For Prostate Brachytherapy. J of Brachy Int. 2000;16:35-43.

21. Lee R. Quality Of Life after Treatment for Localized Prostate Cancer: Differences Based On Treatment Modality. J Clinic Oncol, 2002 Jul; 20(13):3038-3043.

22. Seymore $\mathrm{CH}$, el-Mahdi AM, Schellhammer PF. The effect of prior transurethral resection of the prostate on post radiation urethral strictures and bladder neck contractures. Int J Radiat Oncol Biol Phys. 1986 Sep;12(9):1597-1600.

23. Moran BJ, Stutz MA, Gurel MH.. Prostate brachytherapy can be performed in selected patients after transurethral resection of the prostate. Int J Radiat Oncol Biol Phys. 2004 Jun 1;59(2): 392-396.

24. Stone N, Stock R. Safety and Efficacy of Prostate Brachytherapy in Patients with Prior Turp. Europ Urol 2002 Jan; 1(1):46.

25. Moran BJ, Conterato DJ, Stutz MA, Harris R, Raslowsky MP. Incidence of Urinary Incontinence after I125/Pd103 Prostate Brachytherapy in Patients with Prior Turp. Int $J$ Radiat Oncol Biol Phys 2001 Nov 51; 3(Supp 1):324.

Correspondencia autor: Dr. Daniel Ramirez Claros Departamento de Urología

Hospital dos Servidores do Estado do Rio de Janeiro

Rua Sacadura Cabral, 178 - Saúde - 20221903 Brasil Tel.: 552122913131

E-mail: daniel.ramirezclaros@gmail.com

Información artículo: Original - Cáncer de próstata

Trabajo recibido: octubre 2008

Trabajo aceptado: noviembre 2008 